\title{
A REMARK CONCERNING QUASI-FROBENIUS RINGS
}

\author{
E. A. RUTTER, JR. ${ }^{1}$
}

The purpose of this note is to prove a theorem which establishes a connection between results of [1] and [2]. Recall that for any $R$ module $M$, the "dual" $M^{*}=\operatorname{Hom}_{R}(M, R)$ has a natural structure as a module of the opposite hand from $M$, induced by the bi-module character of $R$.

THEOREM. For any ring $R$, if $\left(\mathrm{a}_{l}\right):$ every $R$-operator homomorphism between minimal left ideals of $R$ is given by a right multiplication, then $\left(\mathrm{b}_{l}\right)$ : the dual of every simple left $R$-module is simple or zero. Conversely, condition $\left(\mathrm{b}_{l}\right)$ implies condition $\left(\mathrm{a}_{l}\right)$, provided that for every minimal left ideal $L$ of $R$, the set $(L)^{0}$ of elements of $R$ which annihilate $L$ on the right is $\neq R$.

The same relationship exists between the analogous conditions $\left(\mathrm{a}_{r}\right)$ and $\left(\mathrm{b}_{r}\right)$ for right ideals and right modules.

In [2, Propositions 1 and 3, pp. 204 and 206], Ikeda proved: If $A$ is an algebra of finite rank containing a left identity [a ring with minimum condition on left and right ideals], then $A$ is quasi-Frobenius if and only if $A$ satisfies $\left(\mathrm{a}_{l}\right)$ [both $\left(\mathrm{a}_{l}\right)$ and $\left.\left(\mathrm{a}_{\mathrm{r}}\right)\right]$. In $[1,(3.4)$ and (4.1), pp. 349 and 350], Dieudonné proved results which are identical in statement to those of [2], cited above in italics, with conditions $\left(a_{l}\right)$ and $\left(a_{r}\right)$ replaced by conditions $\left(b_{l}\right)$ and $\left(b_{r}\right)$, respectively except that he assumed that $A$ had an identity. Our theorem allows immediate passage from Ikeda's results to those of Dieudonné. Used together with Lemma 1 of [2, p. 204], it also allows the reverse passage.

Proof of The Theorem. $(\Rightarrow)$ If $S$ is a simple left $R$-module such that $S^{*} \neq 0, S$ is isomorphic to some minimal left ideal $L$ of $R$ and hence $S^{*} \cong L^{*}$. Let $0 \neq f \in L^{*}$; then $f^{-1}: f(L) \rightarrow L$ exists and is a homomorphism between minimal left ideals of $R$. Consequently, there exists an element $r \in R$ such that $f r=i$ (the identity map on $L$ ). For any $f^{\prime} \in L^{*}$, there is an $r^{\prime} \in R$ such that $f^{\prime}=i r^{\prime}$ and hence $f^{\prime}=i r^{\prime}$ $=(f r) r^{\prime}=f\left(r r^{\prime}\right)$.

$(\Leftarrow)$ Let $L$ be any minimal left ideal of $R$; then $L^{*} \neq 0$ since $i$ belongs to $L^{*}$ and hence $L^{*}$ is simple. Since $(L)^{0} \neq R$ there exists an element $r \in R$ such that $i r \neq 0$ and hence $i R=L^{*}$.

Received by the editors July 3, 1964 .

${ }_{1}^{1}$ This work was done while the author held a NASA Fellowship at Iowa State University. 
REMARKS. (1) It is easy to construct examples of rings which satisfy condition $\left(b_{l}\right)$ but not condition $\left(a_{l}\right)$.

(2) Any ring which satisfies condition $\left(a_{l}\right)$ and contains a minimal left ideal must have $(L)^{0} \neq R$ for every minimal left ideal.

The author wishes to thank Professors B. Vinograde and T. J. Head for their help in organizing this note.

\section{BIBLIOGRAPHY}

1. J. Dieudonne, Remarks on quasi-Frobenius rings, Illinois J. Math. 2 (1958), 346-354.

2. M. Ikeda, A characterization of quasi-Frobenius rings, Osaka Math. J. 4 (1952), 203-209.

IOWA STATE University

\section{ON THE SUBGROUPS OF THE PICARD GROUP'}

HERMANN V. WALDINGER

1. Introduction. The Picard group $\Gamma$ is important in the Theory of Automorphic Functions [3]. It consists of all linear transformations

$$
w=\frac{a z+b}{c z+d}, \quad a d-b c= \pm 1
$$

with coefficients Gaussian integers. $\Gamma$ is known [3] to have four generators

$$
s, t, u, v
$$

together with the eight defining relations

(1.3) $s^{2}=u^{2}=v^{2}=\left(u s^{-1}\right)^{2}=\left(v t^{-1}\right)^{2}=\left(s t^{-1}\right)^{2}=\left(u t^{-1}\right)^{3}=\left(v u^{-1}\right)^{3}=1$.

The generators (1.2) are the transformations $w=-z, w=z-1$, $w=-1 / z$, and $w=-z+i$ respectively.

In this paper, we seek to examine the structure of the Picard group by studying its subgroups. The modular group is a well-known subgroup. It consists of all transformations (1.1) with coefficients

Received by the editors November 19, 1964.

1 This paper was completed under NSF grant GP-3204. 